\title{
Concurrence of Fahr's disease and brain tumor: A case report and review of the literature
}

\author{
QINGTANG LIN ${ }^{1}$, YUESHAN PIAO ${ }^{2}$, FENG LING ${ }^{1}$ and GENG XU ${ }^{1}$ \\ Departments of ${ }^{1}$ Neurosurgery and ${ }^{2}$ Pathology, Xuanwu Hospital, Capital Medical University, Beijing 100054, P.R. China
}

Received October 12, 2014; Accepted September 1, 2015

DOI: 10.3892/etm.2015.2761

\begin{abstract}
Fahr's disease is a rare entity characterized by abnormal calcification in the bilateral basal ganglion regions and cerebellum. Concurrence of Fahr's disease with brain tumor is associated with an even lower incidence; the present study describes the fifth such case, in which a 32-year-old female presented with a 1-month history of headache and unsteady walking. Pathology and imaging studies resulted in a diagnosis of low-grade astrocytoma with calcified deposition. The patient recovered uneventfully. Following a review of the literature, several similarities among the reported cases were revealed. Patients were relatively young at the onset of the disease. The pathological diagnosis for all brain tumors was astrocytoma. The cerebellum was the predominant location of the brain tumor. The imaging studies of these cerebral astrocytomas were almost identical, with a cystic glioma occurring adjacent to a neighboring calcified dentate nucleus. Furthermore, the development of astrocytoma in the bilateral cerebellar hemisphere was not uncommon, which warrants close follow-up for these patients.
\end{abstract}

\section{Introduction}

Fahr's disease is an uncommon disorder characterized by abnormal calcification in the bilateral basal ganglia, thalamus, cerebral cortex and cerebellar dentate nuclei $(1,2)$. The etiology of Fahr's disease remains largely unknown. Certain conditions are implicated in the pathogenesis of Fahr's disease, which include endocrine abnormality, mitochondrial myopathies, dermatological abnormalities and infectious diseases. Clinical manifestations of Fahr's disease consist of a number of symptoms, including tremor, chorea and loss of memory. Treatment strategy focuses primarily on symptom relief and control of accompanying disorders, which are not able to effectively reverse the progression of this disease and thereby improve the

Correspondence to: Dr Qingtang Lin, Department of Neurosurgery, Xuanwu Hospital, Capital Medical University, 45 Changchun Street, Beijing 100054, P.R. China

E-mail: kingsang2002@hotmail.com

Key words: Fahr's disease, brain tumor, glioma, concurrence poor prognosis of the patients. The concurrence of brain tumor with Fahr's disease is even more rare. The fifth such case is described in the present study, and the findings of a review of the literature are discussed. Several intriguing similarities were found among the reported cases.

\section{Case report}

A 32-year-old female patient was admitted to the Department of Neurosurgery at the Xuanwu Hospital (Beijing, China) due to a 1-month history of headache accompanied by unsteady walking. Informed consent was obtained from the patient. Physical examination revealed bilateral papilledema, impaired cerebellar coordination and positive Babinski's sign on the right side. Computed tomography (CT) showed calcification in the bilateral thalamus and dentate nuclei, together with hydrocephalus and a hypo-intense mass in the left cerebellum adjacent to the calcified deposit (Fig. 1A and B). Magnetic resonance imaging (MRI) further demonstrated a non-enhancing cystic space-occupying lesion in the left cerebellar hemisphere (Fig. 2A and B). Thorough laboratory investigations were carried out, which ruled out the possibilities of endocrine abnormalities, viral infection and toxification as the causes of this cerebral calcification (3). Craniotomy via a suboccipital, paramedian approach was performed, during which a cystic astrocytoma containing yellowish fluid and a solid, grayish tumor was completely removed (Fig. 3A and B). Subsequent pathology studies obtained positive result for the proliferation of astrocytes and negative results for angiogenesis and necrosis, which confirmed the lesion as a low-grade astrocytoma intermingled with calcified deposits (Fig. 4). This patient recovered uneventfully and was discharged thereafter, with the alleviation of her symptoms. No further chemotherapy or radiotherapy was administered following the surgical resection. The patient was remained tumor free at the two-year follow-up.

\section{Discussion}

Retrospective review revealed a further 4 documented cases of the concurrence of Fahr's disease with brain tumor (4-6). The median age at diagnosis was 22 years, ranging from 8 to 36 years. There was no gender-specific predisposition ( 3 female vs. 2 male patients). The pathological diagnosis for all cases was low-grade glioma. With regard to the locations 

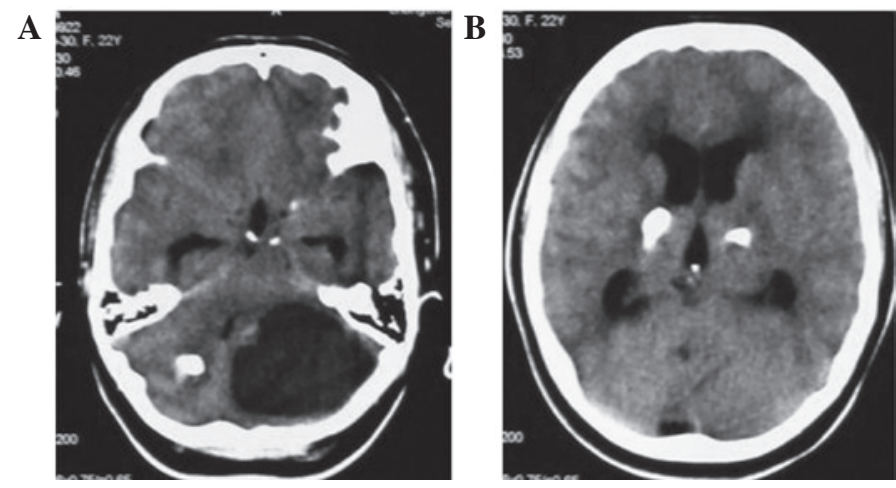

Figure 1. Computed tomography revealed a (A) hypo-intense lesion in the left cerebellum, accompanied by (B) calcification in the bilateral thalamus and dentate nuclei.
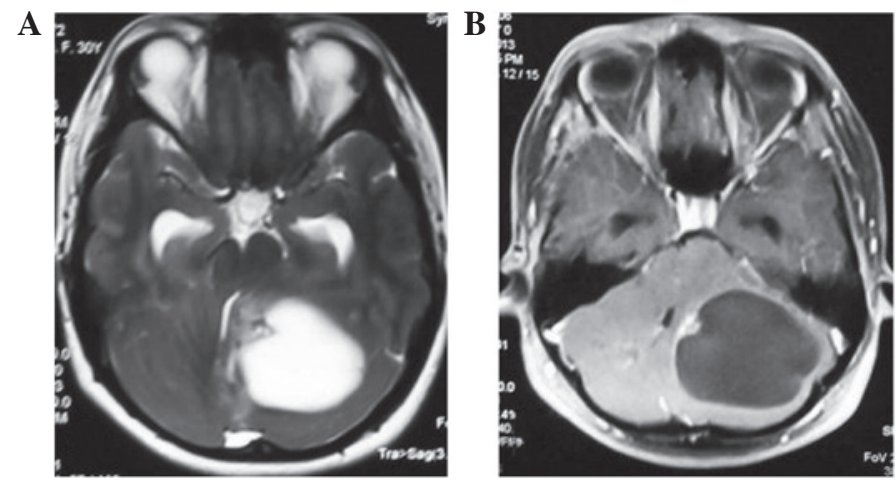

Figure 2. Magnetic resonance imaging showed that this lesion (A) was hyper-intense in the T2 sequence and (B) could not be significantly enhanced.
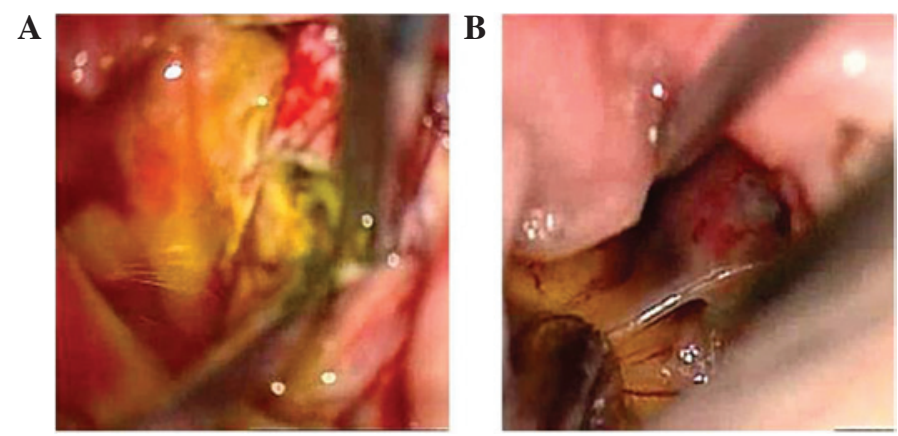

Figure 3. Intraoperatively, (A) the cyst part contained greenish-yellowish fluid, while (B) the solid part appeared to be a reddish tumor with medium blood supply.

A

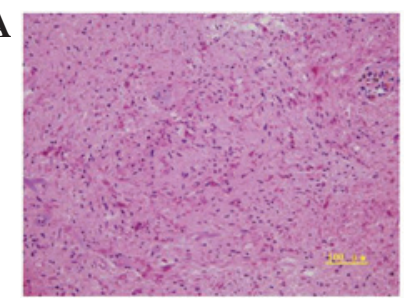

D

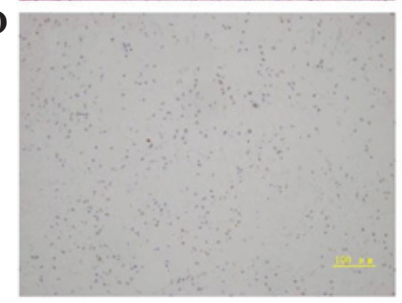

B

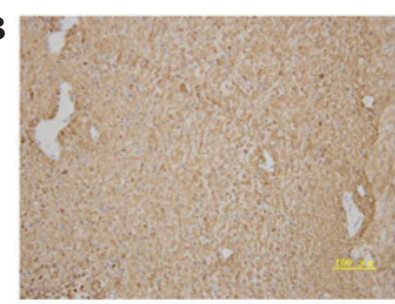

$\mathbf{E}$

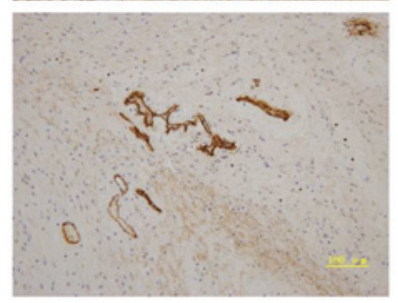

C

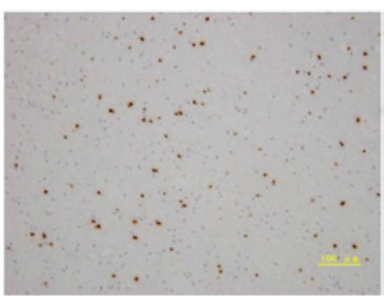

$\mathbf{F}$

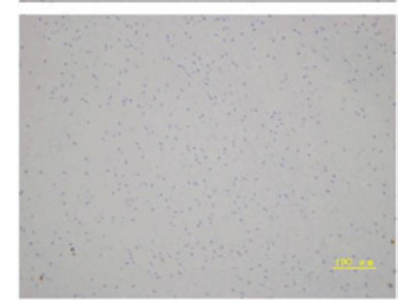

Figure 4. (A) Hematoxylin and eosin staining revealed a glioma intermingled with calcified deposits. (B-F) Immunohistochemistry studies showed that the glioma was positive for (B) glial fibrillary acidic protein, (C) oligo-2, (D) p53 and (E) CD-34, while negative for (F) isocitrate dehydrogenase 1. Scale bar, $100 \mu \mathrm{m}$. 
of the tumors, 4 cases were in the cerebellar hemisphere $(5,6)$ and 1 case was in the parietal lobe (4). Notably, the 4 cases in the cerebellum exhibited similar imaging characteristics. CT revealed calcification in the bilateral basal ganglia, thalamus and cerebellar dentate nuclei, accompanied by hydrocephalus, as shown in the present case (Fig. 1A and B). MRI studies demonstrated that the non-enhancing cystic tumor was always located in the cerebellar hemisphere and adjacent to the neighboring calcified deposit in the dentate nucleus. Furthermore, in 2 of the 4 cases (6), there were two independent cystic gliomas in each cerebellum, which occurred asynchronously and were adjacent to the medial calcified dentate nuclei. The intervals for the development of the other glioma were 3 and 4 years, respectively (6).

To date, the mechanism underlying Fahr's disease remains unclear. Histopathology studies in brain specimens of patients with Fahr's disease have noted extensive calcification throughout the brain tissue, accompanied by hypertrophy and hyperplasia of astrocytes $(3,4)$. It is likely that this astrocytosis contributes to the development of glioma. Furthermore, several genetic alterations that have been implicated in the pathogenesis of Fahr's disease, such as in the platelet-derived grow th factor $\beta$ polypeptide gene, have also been demonstrated by numerous studies to be involved in the development of glioma $(7,8)$. The molecular mechanism underlying the concurrence of these two entities therefore warrants further investigation.

In summary, the diagnosis of brain tumor, particularly low-grade glioma, should be taken into account when patients with Fahr's disease develop symptoms associated with high intracranial pressure or focal neurological dysfunctions. The occurrence of cystic glioma in one cerebellar hemisphere may predispose the other hemisphere to the formation of another cystic glioma, which warrants the close monitoring and follow-up of these patients.

\section{Acknowledgements}

This study was partially supported by the National Natural Science Foundation (grant no. 81101664) and Beijing Natural Science Foundation (grant no. 7132079).

\section{References}

1. Ellie E, Julien J and Ferrer X: Familial idiopathic striopallidodentate calcifications. Neurology 39: 381-385, 1989.

2. Manyam BV, Walters AS and Narla KR: Bilateral striopallidodentate calcinosis: Clinical characteristics of patients seen in a registry. Mov Disord 16: 258-264, 2001.

3. Saleem S, Aslam HM, Anwar M, Anwar S, Saleem M, Saleem A and Rehmani MA: Fahr's syndrome: Literature review of current evidence. Orphanet J Rare Dis 8: 156, 2013.

4. Ang LC, Rozdilsky B, Alport EC and Tchang S: Fahr's disease associated with astrocytic proliferation and astrocytoma. Surg Neurol 39: 365-369, 1993

5. Morimoto K, Mogami H and Ozaki K: Fahr's disease associated with cystic astrocytoma. Eur Neurol 23: 265-268, 1984.

6. Ozerov SS, Semenova ZhB, Zubaŭraev MS, Pitskhelauri DI, Ozerova VI and Strebkova NA: Concurrence of Fahr's disease with cerebellar tumors. Zh Vopr Neirokhir Im N N Burdenko: 34-35, 2004 (In Russian).

7. Nicolas G, Pottier C, Charbonnier C, Guyant-Maréchal L, Le Ber I, Pariente J, Labauge P, Ayrignac X, Defebvre L, Maltête D, et al; French IBGC Study Group: Phenotypic spectrum of probable and genetically-confirmed idiopathic basal ganglia calcification. Brain 136: 3395-3407, 2013.

8. Calzolari F and Malatesta P: Recent insights into PDGF-induced gliomagenesis. Brain Pathol 20: 527-538, 2010. 